SHORT REPORT

\title{
Prospective risk of cancer in CDKN2A germline mutation carriers
}

\author{
A M Goldstein, J P Struewing, M C Fraser, M W Smith, M A Tucker
}

J Med Genet 2004;41:421-424. doi: 10.1136/jmg.2004.019349

\begin{abstract}
Background: The CDKN2A gene is the major known highrisk melanoma susceptibility gene. Susceptibility to other cancers has also been suggested. However, most studies examining the risks of other cancers classified individuals according to the family's CDKN2A mutation rather than determining individual mutation status. For non-populationbased studies, risks could also be biased because of cancer occurrence prior to family ascertainment.

Methods: We examined the risk of non-melanoma cancer in 117 mutation-positive and 136 mutation-negative members from 15 families that had at least two first degree relatives with melanoma and CDKN2A mutations restricting the analysis to the period after the families were ascertained (that is, the prospective period) and using individual mutation data. The families have been followed prospectively for 4-26 years starting in the 1970s.

Results: Overall, there was no significant association for mutation-negative subjects (Obs/Exp $=0.3,95 \%$ confidence interval (CI) 0.0 to 1.2) although this group had only two observed cancers. In contrast, mutation-positive subjects had a significantly increased risk for all cancers combined (Obs/ $\operatorname{Exp}=12 / 5.5=2.2,95 \% \mathrm{Cl} 1.1$ to 3.8 ) primarily because of digestive system tumours, particularly pancreatic cancer. No other organ systems or individual tumour sites showed significantly increased risks.

Conclusions: Differences in CDKN2A-non-melanoma cancer associations across studies may result from variation in genetic backgrounds, insufficient follow up, misclassification of mutation carriers, or the presence of other genetic and/or environmental risk factors in both CDKN2A mutation carriers and non-carriers. Larger sample sizes, prospective follow up, and individual mutation data will be required to understand these differences.
\end{abstract}

$\mathrm{T}$ he $C D K N 2 A$ gene is the major known melanoma susceptibility gene. Germline mutations have been detected in approximately $20 \%$ of melanoma-prone families. $^{12}$ Susceptibility to other cancers has also been suggested. In particular, there is a significantly increased risk of pancreatic cancer in a subset of families with CDKN $2 A$ mutations. $^{3-7}$ And recently, a significantly increased risk of breast cancer was reported in melanoma-prone families with CDKN2A mutations from Sweden. ${ }^{6}$ However, most studies examining the risks of other cancers have not been population-based because of the difficulties in collecting sufficient numbers of families with mutations. In such situations, the selection and ascertainment methods applied to patients/families may influence the associations observed. Specifically, examination of cancer risks may be subject to bias if cancers that occurred prior to ascertainment of the families, that is, cancer occurrence during the retrospective period, influenced participation or selection of families into a research study and such cancer occurrences were included in the risk assessment. In addition, most studies, including our previous study, ${ }^{3}$ classified individuals according to the family's $C D K N 2 A$ mutation rather than determining individual mutation status. ${ }^{3-7}$ This approach could have led to misclassification of both mutation-positive and mutationnegative subjects. These complexities, which could produce biased results, require a more refined approach. We, therefore, examined the risk of non-melanoma cancer in melanoma-prone families with $C D K N 2 A$ mutations restricting the analysis to the period after the families were ascertained (that is, the prospective period) and using individual mutation data.

\section{METHODS}

\section{Participants and design}

Families were recruited for this non-population-based family study if there was a history of invasive melanoma in at least two first degree relatives. The families were referred by health-care professionals or through self-referrals. Written informed consent was obtained prior to participation under an Institutional Review Board-approved protocol. All family members willing to participate in the study were clinically evaluated. Clinical evaluation of family members included complete skin examination and routine medical history. Bloods were collected for genetic studies at the subject's first visit. The subjects for this study were drawn from 15 families in which a CDKN2A mutation had been previously identified. ${ }^{8}$ All the families were Caucasian and resided in various regions of the United States. Table 1 shows the mutation identified, the number of melanoma patients, the number of subjects with known mutation status, and the date of ascertainment in each family. The families have been followed prospectively for 4-26 years starting in the 1970s. Follow up of the families included periodic clinical evaluation and regular requests for updated medical information including the occurrence of cancer. All cancer diagnoses were confirmed by review of histologic materials, local pathology reports, medical records, or death certificates. Only invasive cancers confirmed by at least one of these methods were included in this study.

\section{Statistical analysis}

To estimate the prospective risk of cancer, we calculated numbers of person-years of observation according to sex, age, and the interval from the date of each family's ascertainment to the development of cancer, death, or July 1, 2002. Tumour incidence rates for whites specific for sex, age, and calendar year were obtained from the Surveillance, Epidemiology, and End Results (SEER) program and were multiplied by the total number of person-years to estimate the number of

Abbreviations: $\mathrm{Cl}$, confidence intervals; SIR, standardised incidence ratios 
Table 1 Germline mutations in CDKN2A melanoma-prone families

\begin{tabular}{|c|c|c|c|c|c|c|}
\hline \multirow[b]{2}{*}{ Family } & \multirow{2}{*}{$\begin{array}{l}\text { No. patients } \\
\text { with } \\
\text { melanoma }\end{array}$} & \multirow{2}{*}{$\begin{array}{l}\text { No. subjects with } \\
\text { known CDKN2A } \\
\text { mutation status }\end{array}$} & \multirow{2}{*}{$\begin{array}{l}\text { Date of } \\
\text { ascertainment of } \\
\text { family }\end{array}$} & \multicolumn{3}{|c|}{ Description of CDKN2A mutation } \\
\hline & & & & Exon & $\begin{array}{l}\text { Amino acid } \\
\text { change }\end{array}$ & $\begin{array}{l}\text { Nucleotide } \\
\text { change }\end{array}$ \\
\hline A & 8 & 32 & 1976 & $1 \alpha$ & 1_8dup8 & 24_47dup24 \\
\hline B & $6^{*}$ & 10 & 1994 & $1 \alpha$ & L16R & $47 \mathrm{~T}>\mathrm{G}$ \\
\hline C & 5 & 13 & 1995 & 2 & M53I & $159 G>C$ \\
\hline D & $7^{*}$ & 19 & 1979 & 2 & A58X & $172 C>T$ \\
\hline $\mathrm{E}$ & $4^{*}$ & 15 & 1979 & 2 & N71S & $212 A>G$ \\
\hline $\mathrm{F}$ & $12^{*}$ & 23 & 1979 & 2 & R87P & $260 G>C$ \\
\hline G & 5 & 12 & 1979 & 2 & G101W & $301 \mathrm{G}>\mathrm{T}$ \\
\hline $\mathrm{H}$ & 3 & 7 & 1979 & 2 & G101W & $301 G>T$ \\
\hline I & $5^{*}$ & 8 & 1988 & 2 & G101W & $301 \mathrm{G}>\mathrm{T}$ \\
\hline J & 7 & 11 & 1980 & 2 & V126D & $377 T>A$ \\
\hline K & $6^{*}$ & 11 & 1979 & 2 & V126D & $377 \mathrm{~T}>\mathrm{A}$ \\
\hline $\mathrm{L}$ & 10 & 33 & 1998 & 2 & V126D & $377 T>A$ \\
\hline $\mathrm{N}$ & $8^{*}, *$ & 10 & 1992 & 2 & S56fst & 167_197del31 \\
\hline$P$ & 11 & 45 & 1994 & 2 & Chimerał & 240_253del14 \\
\hline$Q$ & 3 & 4 & 1988 & Intron 2 & Splicing§ & $+\lg >t$ \\
\hline
\end{tabular}

occurrences of cancer expected if this group had had the same risk of cancer as the general population. For 1999-2002, 1998 incidence rates were used. ${ }^{10}$ Tumours diagnosed before each family was ascertained were excluded, that is, only incident cancers that occurred after ascertainment of each family were included in the analysis. Tests of significance and 95\% confidence intervals (CI) for the standardised incidence ratios (the ratio of the number of observed cancer occurrences to the number expected) were calculated exactly on the basis of a Poisson distribution. Standardised incidence ratios (SIR) were calculated separately for subjects who were mutation-positive (with and without melanoma) and mutation-negative. All tests were two-sided.

\section{RESULTS}

Only bloodline subjects with known mutation status were included; $84 \%$ of bloodline subjects with DNA had been mutation-tested $(n=210)$ or genotyped to determine if they carried their family's disease-specific haplotype $(\mathrm{n}=43)$. Thus, there were 253 subjects of whom 117 were CDKN2A mutation-positive and 136 mutation-negative. Of the 117 mutation-positive subjects, 64 had invasive or in situ melanoma. Of mutation-negative subjects $95 \%$ were first degree $(n=76)$ or second degree $(n=53)$ relatives of mutation-positive individuals. The remaining seven mutationnegative subjects were third degree relatives of mutationpositive individuals. Table $2 \mathrm{~A}$ presents the prospective risks of cancers in mutation-positive and mutation-negative subjects. Cancers were considered according to organ system rather than individual site (for one or fewer cancers) because of the relatively small numbers. Table 3 presents details for the 14 reported prospective non-melanoma cancers. Overall, there was no significant association for mutation-negative subjects ( $\mathrm{SIR}=0.3,95 \%$ CI 0.0 to 1.2 ), although this group had few observed cancers. In contrast, mutation-positive subjects had a significantly increased risk for all cancers combined ( $\mathrm{SIR}=2.2,95 \%$ CI 1.1 to 3.8 ) primarily because of digestive system tumours, particularly pancreatic cancer (table 2A). Four patients with pancreatic cancer from three different families (families F, J, K) were observed ( SIR $=38,95 \%$ CI 10 to 97). There were no occurrences of pancreatic cancer in mutation-negative subjects. No other organ systems or individual tumour sites showed significantly increased risks.

Mutation-positive subjects were further split into those with and without invasive or in situ melanoma (table 2B). Nine of the 12 prospective cancers from mutation-positive subjects occurred in patients with melanoma. And as was previously seen, there were significantly increased risks for all cancers combined ( $\mathrm{SIR}=2.3,95 \%$ CI 1.1 to 4.4 ), digestive system tumours ( $\operatorname{SIR}=9,95 \%$ CI 3 to 20 ), and pancreatic cancer $(S I R=52,95 \%$ CI 14 to 133$)$. The relatively small numbers of cancers, however, yielded imprecise estimates of the prospective cancer risks. Among subjects without melanoma, there were no significant associations; three cancers were observed ( SIR $=1.9,95 \%$ CI 0.4 to 5.5 ), two of which were breast cancer ( SIR $=5.5,95 \%$ CI 0.6 to 20.0) from different families (table 3).

Although pancreatic cancer showed a significantly increased risk in mutation-positive subjects, during this prospective follow-up period, only four patients developed pancreatic cancer based on 1500 person-years of observation. In contrast, during the same prospective period, 49 invasive melanomas developed in 22 melanoma patients, including seven patients who developed their first melanoma. In addition, there were 14 deaths related to melanoma during this prospective period. Thus, melanoma remains the major contributor to morbidity and mortality in these subjects. And, although melanoma has a dramatically earlier age at diagnosis in melanoma-prone families with CDKN2A mutations, ${ }^{12}$ the median age at pancreatic cancer diagnosis in this study (70.5 years) was consistent with that observed in the US general population (median 71.0 years). ${ }^{10}$

\section{DISCUSSION}

Previous studies have reported significantly increased risks for non-melanoma cancers, especially pancreatic cancer and rarely breast cancer, in $C D K N 2 A$ mutation-positive melanomaprone families. ${ }^{13-7}$ Most of these studies, however, did not use mutation data from individual participants and therefore may have misclassified both mutation-positive and mutation-negative subjects. ${ }^{3-7}$ In addition, some of these studies were not population-based and so the occurrence of other cancers in the families might have influenced participation or selection of families into a research study. Under this scenario, assessment of cancer risk that included cancers that had occurred in the families prior to ascertainment could have biased the results. To eliminate this potential bias, evaluation of cancer risks from non-population-based family studies should be restricted to the period after ascertainment of the families, if possible. However, this approach requires prospective follow up of the families; such follow up may not be feasible for many study samples. This non-populationbased family study tried to eliminate both of these potential problems by restricting the statistical analysis to the 
Table 2 Prospective risk of non-melanoma cancer in CDKN2A melanoma-prone families

\begin{tabular}{|c|c|c|c|c|c|c|c|c|}
\hline \multicolumn{9}{|c|}{ A. CDKN2A mutation-positive and mutation-negative subjects } \\
\hline \multirow[b]{2}{*}{ Tumour type } & Obs & Exp & Obs/Exp & $(95 \% \mathrm{Cl})$ & Obs & Exp & Obs/Exp & $(95 \% \mathrm{Cl})$ \\
\hline & \multicolumn{4}{|c|}{ Mutation-positive subjects (1500 person-years) } & \multicolumn{4}{|c|}{ Mutation-negative subjects (2138 person-years) } \\
\hline All cancers & 12 & 5.5 & 2.2 & $(1.1$ to 3.8$)$ & 2 & 6.2 & 0.3 & $(0.0$ to 1.2$)$ \\
\hline Digestive system & 6 & 0.9 & 6.7 & (2.4 to 14.6 ) & 0 & & & \\
\hline Pancreas & 4 & 0.1 & 38 & (10 to 97$)$ & 0 & & & \\
\hline Respiratory system & 1 & 0.8 & 1.2 & (0.0 to 6.5$)$ & 0 & & & \\
\hline Breast & 2 & 0.9 & 2.2 & $(0.2$ to 8.1$)$ & 1 & 1.2 & 0.9 & $(0.0$ to 4.8$)$ \\
\hline Prostate & 1 & 0.8 & 1.3 & $(0.0$ to 7.4$)$ & 0 & & & \\
\hline Urinary tract & 1 & 0.4 & 2.6 & $(0.0$ to 14.6$)$ & 0 & & & \\
\hline Brain and CNS & 1 & 0.1 & 10.6 & (0.1 to 59.1$)$ & 0 & & & \\
\hline Lymphatic and haematopoietic & 0 & & & & 1 & 0.6 & 1.8 & (0.0 to 9.8$)$ \\
\hline \multicolumn{9}{|c|}{ B. CDKN2A mutation-positive subjects only } \\
\hline \multirow[b]{2}{*}{ Tumour type } & Obs & Exp & Obs/Exp & $(95 \% \mathrm{Cl})$ & Obs & Exp & Obs/Exp & $(95 \% \mathrm{Cl})$ \\
\hline & \multicolumn{4}{|c|}{ Subjects with melanoma (811 person-years) } & \multicolumn{4}{|c|}{ Subjects without melanoma (689 person-years) } \\
\hline All cancers & 9 & 3.9 & 2.3 & $(1.1$ to 4.4$)$ & 3 & 1.6 & 1.9 & $(0.4-5.5)$ \\
\hline Digestive system & 6 & 0.7 & 9.1 & (3.3 to 19.8 ) & 0 & & & \\
\hline Pancreas & 4 & 0.1 & 52 & (13 to 132$)$ & 0 & & & \\
\hline Respiratory system & 1 & 0.7 & 1.5 & $(0.0$ to 8.5$)$ & 0 & & & \\
\hline Breast & 0 & 0.5 & & $(0.0$ to 6.9$)$ & 2 & 0.4 & 5.5 & $(0.6-20.0)$ \\
\hline Prostate & 1 & 0.6 & 1.6 & $(0.0$ to 8.8$)$ & 0 & & & \\
\hline Urinary tract & 1 & 0.3 & 3.4 & $(0.0$ to 19.0$)$ & 0 & & & \\
\hline Brain and CNS & 0 & 0.1 & & (0.0 to 58.4$)$ & 1 & 0.03 & 31.9 & $(0.4-177.3)$ \\
\hline
\end{tabular}

prospective time period, that is, after the date of ascertainment, and by using individual mutation data. Although based on small numbers, the results suggested that the major increased risk for non-melanoma cancers in these melanoma-prone families with CDKN2A mutations resulted from an increased risk of digestive system tumours, primarily pancreatic cancer. At present, however, we cannot identify the specific genotypes that predispose individuals with a $C D K N 2 A$ mutation to pancreatic cancer. ${ }^{3-7}$ And in contrast to the Swedish study of predominantly one single CDKN2A founder mutation (113insR), ${ }^{6}$ no statistically significantly increased risk for breast cancer was seen here although the trend showed the same direction. Additional studies with much larger sample sizes are required to determine whether specific CDKN2A mutations are associated with different types of cancer.

The families for the current study were ascertained through health care professionals or through self-referrals and may not be representative of all melanoma-prone families with $C D K N 2 A$ mutations. In addition, the occurrence of only 14 prospective non-melanoma cancers precluded a more rigorous statistical analysis. All subjects were treated as independent observations and the 11 CDKN2A mutations were classified identically. Finally, although not all cancers may have been reported, our regular contact with and follow up of the participants should have limited the chances of missing cancer diagnoses. To further minimise errors, only cancers that could be confirmed through review of histologic materials, local pathology reports, medical records, or death certificates were included in this study.

In summary, evaluation of the prospective risk of cancer in $117 C D K N 2 A$ mutation-positive participants showed that the major increased non-melanoma cancer risk resulted from digestive system tumours, primarily pancreatic cancer. However, only four patients developed pancreatic cancer based on 1500 person-years of observation compared to 49 prospective invasive melanomas in 22 melanoma patients and 14 deaths related to melanoma during this prospective period. Thus, melanoma remains the major contributor to morbidity and mortality in these subjects. Differences in

Table 3 Prospective non-melanoma cancers in study participants

\begin{tabular}{cllll}
\hline Subject no. & Tumour type/organ & Histopathology & Age at diagnosis & Family \\
\hline 1 & Lung & Squamous carcinoma & 78 & $\mathrm{~A}$ \\
2 & Lymphatic/haematopoietic & Multiple myeloma & 62 & $\mathrm{~A}$ \\
3 & Prostate & Adenocarcinoma & 50 & $\mathrm{E}$ \\
4 & Brain & Glioblastoma multiforme & 60 & $\mathrm{E}$ \\
5 & Colorectal & Adenocarcinoma & 41 & $\mathrm{~F}$ \\
6 & Pancreas & Carcinoma & 56 & $\mathrm{~F}$ \\
7 & Colorectal & Adenocarcinoma & 38 & $\mathrm{G}$ \\
8 & Pancreas & Carcinoma & 67 & $\mathrm{~J}$ \\
9 & Kidney & Renal cell carcinoma & 55 & $\mathrm{~K}$ \\
10 & Pancreas & Adenocarcinoma & 76 & $\mathrm{~K}$ \\
11 & Pancreas & Carcinoma & 74 & $\mathrm{~K}$ \\
12 & Breast & Infiltrating ductal carcinoma & 41 & $\mathrm{~K}$ \\
13 & Breast & Infiltrating ductal and lobular & 74 & \\
14 & carcinoma & & \\
\hline
\end{tabular}


CDKN2A-non-melanoma cancer associations across studies may result from bias resulting from inclusion of cancers that occurred during the retrospective period (from nonpopulation-based studies), misclassification of mutation carriers, insufficient/low power, variation in the underlying genetic background of families (for example different $C D K N 2 A$ mutations), or the presence of other genetic and/or environmental risk factors in both CDKN2A mutation carriers and non-carriers. Larger numbers of individuals and families with a broad spectrum of mutations, sufficient person-years of prospective follow up, and individual mutation data will be required to better understand differences in these associations.

\section{ACKNOWLEDGEMENTS}

We are indebted to the participating families, whose generosity and cooperation have made this study possible. The authors also wish to acknowledge the contributions to this work that were made by Laura Fontaine, BSN, Virginia Pichler, RN PhD, and Deborah Zametkin, MSN. We would like to thank Melissa Levasseur for genotyping some of the samples used in this work and Joe Barker, IMS, for analytic support.

\section{Authors' affiliations}

A M Goldstein, M C Fraser, M A Tucker, Genetic Epidemiology Branch, Division of Cancer Epidemiology and Genetics, National Cancer Institute, NIH, DHHS, Bethesda, MD 20892, USA

J P Struewing, Laboratory of Population Genetics, Center for Cancer Research, National Cancer Institute, NIH, DHHS, Bethesda, MD 20892, USA

M W Smith, Laboratory of Genomic Diversity, Center for Cancer Research and Basic Research Program, SAIC Frederick, National Cancer Institute at Frederick, NIH, DHHS, Frederick, MD 21702, USA

This publication has been funded in part with funds from the National Cancer Institute, NIH, DHHS, Contract No. NOl-CO-12400.

Conflict of interest: none declared.
Correspondence to: Dr A M Goldstein, Genetic Epidemiology Branch/ $\mathrm{NCl} / \mathrm{NIH} / \mathrm{DHHS}$, Executive Plaza South, Room 7004, 6120 Executive Blvd., MSC 7236, Bethesda, MD 20892-7236, USA; goldstea@ exchange.nih.gov

Revised version received 8 March 2004

Accepted for publication 9 March 2004

\section{REFERENCES}

1 Kefford RF, Newton-Bishop JA, Bergman W, Tucker MA, on Behalf of the Melanoma Genetics Consortium. Counseling and DNA testing for individuals perceived to be genetically predisposed to melanoma: a consensus statement of the Melanoma Genetics Consortium. J Clin Oncol 1999;17:3245-51.

2 Goldstein AM, Tucker MA. Review: genetic epidemiology of cutaneous melanoma. A global perspective. Arch Dermatol 2001;137:1493-6.

3 Goldstein AM, Fraser MC, Struewing JP, Hussussian CJ, Ranade K, Zametkin DP, Fontaine LS, Organic SM, Dracopoli NC, Clark WH Jr, Tucker MA. Increased risk of pancreatic cancer in melanoma-prone kindreds with pl6 $6^{\text {ink4 }}$ mutations. N Engl J Med 1995;333:970-4.

4 Bergman W, Gruis N. Correspondence: familial melanoma and pancreatic cancer. N Engl J Med 1996;334:471.

5 Ghiorzo P, Ciotti P, Mantelli M, Heouaine A, Queirolo P, Rainero ML, Ferrari C, Santi PL, De Marchi R, Farris A, Ajmar F, Bruzzi P, BianchiScarra G. Characterization of Ligurian melanoma families and risk of occurrence of other neoplasia. Int J Cancer 1999;83:441-8.

6 Borg A, Sandberg T, Nilsson K, Johannsson O, Klinker M, Masback A, Westerdahl J, Olsson $\mathrm{H}$, Ingvar $\mathrm{C}$. High frequency of multiple melanomas and breast and pancreas carcinomas in CDKN2A mutation-positive melanoma families. J Natl Cancer Inst 2000;92:1260-6.

7 Vasen HFA, Gruis NA, Frants RR, van der Velden PA, Hille ETM, Bergman W. Risk of developing pancreatic cancer in families with familial atypical multiple mole melanoma associated with a specific 19 deletion of pl6 (p16-Leiden). Int J Cancer 2000;87:809-11.

8 Goldstein AM, Struewing JP, Chidambaram A, Fraser MC, Tucker MA. Genotype-phenotype relationships in U.S. melanoma-prone families with CDKN2A and CDK4 mutations. J Natl Cancer Inst 2000;92:1006-10.

9 Rutter JL, Goldstein AM, Davila MR, Tucker MA, Struewing JP. CDKN2A point mutations D153spl(c. 457G $>$ T) and IVS2+1G $>$ T result in aberrant splice products affecting both pl $6^{\text {ink4a }}$ and $\mathrm{pl} 4^{\text {ARF }}$. Oncogene 2003;22:4444-8.

10 Surveillance, Epidemiology, and End Results (SEER) Program (www.seer.cancer.gov) Public-Use Data (1973-1999), NCI, DCCPS Surveillance Research Program, Cancer Statistics Branch, released April 2002, based on the November 2001 submission. 